\title{
Innovative Solutions for the Automatic Payment of Car Parks
}

\author{
Giuliano Benelli, Alessandro Pozzebon \\ University of Siena, Italy
}

\begin{abstract}
In this paper we describe a solution for the payment of car parks based on different telecommunication technologies. The solution focuses both on street car parks, i.e. parks located along the streets or in the squares, and on closed car parks, $i$. e. parking areas and infrastructures enclosed by entrance and exit bars.

This system has been studied in order to reduce the timing of payment operations and to improve the accuracy of parking fares and is based on different technological solutions using the following technologies: the GPS localization and GPRS connectivity, the UMTS connectivity on mobile phones, the RFID technology and the new short range Near Field Communication (NFC) telecommunication technology. The proposed system allows the user to park the car and then to communicate the time of the parking to a server in an automatic way. At the moment of car picking the user communicates the exact hour and the exact fare is calculated and automatically withdrawn. Together with the application for the user, also a mobile solution for traffic officers has been studied, to allow them to check whether someone is paying or not for a parked car, and an Internet site has been developed, to allow the users to interact with the system.
\end{abstract}

\section{Introduction}

The number of cars circulating in European cities is increasing year after year: while the carinhabitants ratio in Italy is the highest in Europe (around 60 cars for 100 inhabitants) in the most part of European countries this ratio is around 50 cars every 100 inhabitants. Together with this value also the number of car parks is growing, not only in the big cities but also in mid-sized centers and even in small towns which present significant tourist fluxes.

The growth of car parks doesn't concern only the central areas of the cities but also the outskirts where new park and ride structures have been realized, allowing people who don't want to drive to the city center to park their cars and then use the public transport. Moreover, in bigger cities all the car parks are provided with a fee, to safeguard the right of the residents to park their cars and to discourage the use of cars instead of public transport.

Just as an example we can cite the city of Florence in Italy, where in the whole metropolitan area the car parks are with a fee or reserved for the residents.

Fee car parks can be divided in two different categories: closed car parks and street car parks. Closed car parks are usually multilevel structures where cars drive in taking a ticket from a ticketing machine positioned next to an entrance bar which opens once the user has picked the ticket. For these structures the operative protocol follows these steps:

- The user arrives at the entrance bar and receives a ticket with the entrance hour printed on it;

- The entrance bar opens and the user can park the car wherever he wants inside the parking;

- When the user comes back to the parking, before collecting the car, he has to go to a ticketing machine;

- at the ticketing machine he introduces the ticket, and the exact fare is calculated;

- The user introduces the required amount and the ticket is marked as "Paid";

- The user can now pick up the car and go to the exit bar;

- Here he introduces the ticket that, once read, allows the opening of the bar.

This protocol has two key points:

- The timing. The printing and reading operations at the bars introduce a delay, while the user also loses time to go to the ticketing machine. Moreover, in peak hours queues may appear forcing the user to wait for its turn;

- The payment. Few machines provide the Credit Card payment option: this means that the user has to pay using either coins or banknotes.

On the other hand, street car parks are located along the streets and in the squares and don't present an entrance bar: in this case it becomes then more difficult to check whether a user is paying or not for the parking. The payment protocol for these structures is usually based on the following steps:

- The user parks his car wherever he wants;

- Once he has parked the car, he goes to the nearest ticketing machine;

- He selects the amount of time he wants to let the car in the parking;

- He pays the required amount and he receives a ticket showing the ending time of his permanence; 
- He puts the ticket on the dashboard of the car taking care to leave it in a place where it can be seen from the outside.

While this protocol is currently used in most part of European cities, it presents several key factors affecting the user. The following key factors can be underlined:

- The timing. The protocol requires in fact some minutes: the user has to find the ticketing machine that can be in some cases some hundreds of meters away from the car, sometimes he has to queue, and he has to wait for the printing of the ticket.

- The payment. Many ticketing machines only accept coins, making the payment difficult for users who want to pay for many hours, while some other machines don't give the change.

- The extension or the reduction of the parking time. If the user wants to leave the car in the parking for an additional span of time he has to come back to the ticketing machine and pay again while if he picks up the car in advance he cannot get back the amount of money paid in advance.

The actual protocol is instead a good solution for the parking authority: in this case the parking operator simply reads the ending time written on the tickets positioned on the dashboard of all the cars parked in the parking area.

\section{The Proposed Solution}

All the points listed before for the two scenarios can be solved using different technological means. For closed car parks the solution requires a contactless user identification at the entrance and exit bars, together with a remote payment system: in this case the user has not to go to the ticketing machine and he saves time both during the payment and entrance and exit operations.

In this case the proposed protocol follows these steps:

- The user enters the parking using a contactless authentication system that is used also to identify him;

- He parks the car wherever he wants inside the parking;

- When he comes back to the parking he collects the car and goes to the exit bar;

- At the exit bar he is identified again and the exact amount is calculated;

- The amount is the remotely withdrawn and the exit bar opens.

For street car parks the user has to estimate an approximate parking period: this is usually done rounding the total up, and the user in most cases pays for a permanence time that he doesn't enjoys. If the user could indicate the arriving time without paying in advance and then pay only at the end of the parking period, the calculation of the due amount could evidently be done on the exact permanence time. However, to do so the user should have to communicate in some way the beginning of his permanence to parking authority, and this cannot evidently be done with a ticket positioned on the dashboard of the car: evil-minded users could in fact pick the ticket, position it on the dashboard and then, once back to the car, drive away without paying.

The only way to avoid this eventuality is to perform remotely the payment of the parking in automatic way.

The starting moment of the parking will be then communicated to a remote data center which will also record the ending time: in this way the user is forced to communicate when he will pick the car because otherwise he will continue to pay for the parking.

The protocol we propose is based on the following steps:

- The user parks his car wherever he wants;

- Once he has parked the car, he communicates the starting time to the remote data center through one of the three technologies that will be described later;

- When the user comes back at the car he communicates in the same way the ending time.

The payment is performed separately from the parking operation. It can be performed in many ways, for example using the Credit Card or by Bank Transfer, and it can be done after every parking or even once a month. The system can keep trace of every permanence of the user, allowing several operations, like periodic checks on the payment operations or statistic analysis on the habits of the users.

For street car parks the modification of the protocol for the parking use, also requires a change in the protocol for the parking operator charged to check whether a car is paying or not for the parking. As already described, currently the operator simply verifies the presence and the validity of the ticket. With the proposed system the paper ticket is going to disappear, forcing them to find a new check procedure. The proposed solution foresees the use of PDAs (Personal Digital Assistants) for the operators: every PDA has to be provided with an ad-hoc software that will be described later. The PDA is directly connected with the Remote Data Center: the operator can then check whether a car is paying or not simply introducing the registration number of the car in the PDA application, that will then check its presence inside the data center.

For all the solutions the remote data center has been realized using a server PC with a simple MySQL database for all the data concerning the 
parking and with a simple Java application for the calculation of parking fees.

\section{The Closed Car Parks Applications}

Two different solutions have been developed for closed car parks: these solutions foresee the use of two different technologies, the RFID and the NFC technology, even if the applicative protocol is very similar.

While the NFC solution has been developed before, its possible use is currently limited by the low diffusion of NFC devices. This limitation has led to the development of the RFID solution doesn't require an ad-hoc end user device and is then easier to be implemented.

\section{A. The NFC solution}

Near Field Communication represents one of the most interesting telecommunication technologies appeared in the last years [1], [2]. NFC technology is a direct evolution of RFID (Radio Frequency IDentification) automatic identification system in that is a short range (up to $10 \mathrm{~cm}$ ) wireless communication channel operating at the unregulated frequency of $13,56 \mathrm{MHz}$. NFC technology has been studied to be embedded on mobile phones, allowing these devices alternately to read contactless smart cards, to be read by ad-hoc readers as common smart cards or simply the exchange data among them. NFC has been studied mainly to allow mobile payment and mobile ticketing applications, where the phone can be used as an electronic wallet, as a contactless credit card or as an electronic ticket. This means that NFC is the perfect solution for a scenario where contactless ticketing and payment are required.

The proposed solution is based on the use of an NFC phone as the mean to access to the parking and then as the payment device at the exit [3]. Together with the NFC phones the following devices have been used for the development of the prototype:

- An NFC Reader simulating the Entrance bar;

- An NFC Reader simulating the Exit bar;

- A kiosk where the user can upload the virtual money on his NFC phone.

Three applications together with the underlying applicative protocols compose the software infrastructure of the system.

The first one is the Entrance application: this software manages then access of the user to the parking area. The main purpose of this application of to communicate with the reader positioned next to the Entrance bar of the parking. In particular, this applicative protocol follows these steps:
- The user arrives at the entrance bar with his car and brings his NFC phone close to the Reader;

- The Reader reads the ID code of the NFC phone and identifies the user;

- Once the user is identified, a Record is created in the remote database storing the parking data, marking the user has Inside the parking and recording the entrance timing;

- The Entrance bar opens and the user can park his car;

The second application manages the Exit operations. In this case the applicative protocol is a bit more complex in that also involves the payment, and is based on these steps:

- The user arrives at the Exit bar with his car and identifies himself allowing his phone to be read by the NFC reader positioned at the Exit bar;

- The application searches inside the database for the record associated with the user where he is marked as Inside the parking;

- The exact fare is calculated using the Entrance time stored inside the database and the exact Exit time;

- The system check whether the virtual amount stored inside the internal NFC memory of the phone is sufficient to pay for the parking;

- If the virtual money stored inside the phone is enough, the amount of the parking is withdrawn and the exit bar opens;

- If the virtual money is not sufficient the user is invited to go to the kiosk to upload new virtual money.

The last application is deployed on the kiosk and allows the user to upload virtual money on the internal memory of his NFC phone. In this case a simple Touch Screen interface is provided, that allows the user to select the amount he wants to upload. The money can be introduced using ad-hoc cash or Credit Card slots. Once the money is introduced, the user simply brings his phone close to the NFC reader embedded inside the kiosk and the amount is uploaded. The user can check the effective money upload using a Check your Credit function.

\section{B. The RFID solution}

The RFID solution has been developed in order to overcome the limitations due to the low level of worldwide diffusion of NFC devices [?]. While the number of NFC-enabled mobile phones currently on the market is still notably low, the necessity for systems making easier and faster the parking operations is pressing, and this means that alternative solutions have to be studied and developed. 
The use the RFID automatic identification technology offers the chance to develop an application provided with a total compatibility with the NFC solution. As already underlined, NFC is in fact a direct evolution of the RFID systems, and it retains a quite total level of compatibility with this technology. NFC devices, when used in passive mode as for example electronic wallets or identification devices, simply act as RFID Smart Cards. In particular, NFC devices are complain with the ISO14443 standard for High Frequency $13.56 \mathrm{MHz}$ RFID systems: systems operating at this frequency and compliant with this standard can operate both with RFID Smart Cards and NFC devices. The solution for the closed car parks systems proposes to provide the users with a common ISO14443 RFID Contactless Smart Card providing the same functionalities of an NFC phone. RFID Smart Cards can store data up to 4kbytes, and are provided with a unique ID Code that allows their use as identification devices.

In the proposed solution the RFID card works both as the identification device for the user and as the electronic wallet storing the virtual amount of money inside its internal memory. In this case the applicative protocols are quite the same are the ones proposed for the previous solutions: the only difference derives from the fact that the NFC phone is substituted by the Smart Card in all its functions. No additional change is required to the other components of the system.

\section{The Street Car Parks Applications}

For street car four different solutions have been studied to allow users to communicate the start and end times of the parking, in order to provide them with different choices. The first two solutions are similar to the ones proposed for the closed car parks and are based on the use of the Near Field Communication and RFID technologies.

The third solution uses the mobile phones of the users and has been thought mainly for people owning a Smart Phone: in particular the solution has been customized for the Windows Mobile and iPhone OS platforms.

The fourth solution points to users who don't want to use their mobile phone and is based on the use of an adhoc device provided with a GPS positioning system and communicating through the GPRS channel.

\section{A. The NFC and the RFID solutions}

NFC represents a good solution also to pay street car parks. In this case the NFC phone of the user doesn't act as an electronic key to allow the user to enter the parking, but only as an electronic ticket and an electronic wallet.
The protocol for this solution follows these steps:

- The user parks the car;

- The user goes to the ticketing machine and brings the phone next an NFC reader directly embedded in the machine;

- The machine reads the NFC code stored inside the Secure Element of the phone NFC circuitry;

- The machine sends a message to the data center communicating the code;

- The data center looks for the registration number of the car associated with the NFC code and writes the arrival time;

- When the user comes back he goes to the ticketing machine and brings the phone close to the reader.

- The machine communicates again the code to the data center, and the exact fare is calculated;

- The fare can be withdrawn from the bank account of the user or can also be communicated to the ticketing machine. In this case the fare can be directly withdrawnfrom a virtual credit stored inside the Secure Element of the NFC phone of the user [7].

The proposed solution has the only drawback that the user is forced to go to the ticketing machine of the parking at the arrival and at the departure: anyway, the transaction times are very fast and in practice the user has only to arrive at the machine and bring the phone next to the area where an embedded NFC reader is located: the reading times are usually lower than one second.

As in the previous case, this solution is currently delayed by the slow diffusion of NFC phones. Anyway, also in this case the system can be adapted to work with exactly the same features with ISO14443 compliant RFID Smart Cards. As for closed car parks the only modification to the applicative protocol derives from the fact that all the operations requiring the NFC device are performed with the RFID card. This solutions will boost the rapid diffusion of such a kind of systems: the compatibility between the two technologies will allow in fact the extension of the functionalities of the system to the NFC devices without the need for any change to the hardware devices and to the software infrastructure of the data center.

\section{B. The Mobile Solution}

While applications for the payment of the parking through SMSs have already been studied and developed in many parts of the world [5], [6], we propose a solution using the UMTS channel of the phone: in particular, we suggest the installation of an ad hoc application on the phone of the user to 
indicate the data center the place and the time of the parking.

For this solution the protocol is based on the following steps:

- The user parks the car;

- After he has parked the car he opens the application on his mobile phone;

- He selects the parking area where he has left the car from a scroll list and he introduces the registration number of the car. The already entered numbers can be chosen from a scroll list;

- He presses an "Enter" button and this information is sent to the data center using the UMTS communication channel of the mobile phone. The application simply performs an HTTP POST;

- $\quad$ Once back to the car, the user applies on his phone the same procedure followed when he parked the car;

- The fare is then calculated by the data center and then withdrawn.

This solution is very simple. The interaction with the mobile phone is limited and the user can do this operation even in motion while walking away from the car.

Moreover, not requiring additional devices, this solution is virtually costless. The software application has been developed for only two platforms (Windows Mobile and iPhone OS), but can be easily extended also to all the other mobile platforms (Android, Blackberry, Sybian, etc...).

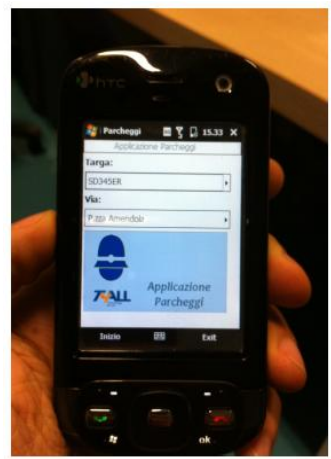

Figure 1. The Windows application

\section{The GPS Solution}

The fourth solution is the only one that uses an adhoc device, which has the main purpose to communicate the place where the car is parked and the parking hours. The device is a small black box whose dimensions are around $8 \times 8 \times 3.5 \mathrm{~cm}$, and on the outside it presents only a red button, a small reset button and a LED indicating if the box is on.

Inboard the box houses an XT65 Siemens Cellular Engine: this device integrates into a single platform a GPRS communication module, a GPS localization system, a microprocessor provided with a Java Virtual Machine and a RS232 serial communication port.

The operative protocol for this solution is very simple and follows these steps:

- The user parks the car;

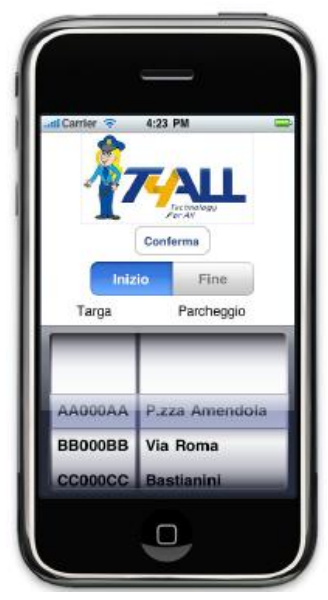

Figure 2. The iPhone application

- Before he leaves the car he presses the red button on the box: at this moment the device communicates, through the GPRS module, the position of the car calculated by the GPS module and the registration number of the car. These data are used to calculate the fare. In particular the position is used to determine where the car is parked and which is the fare of associated to that area; the registration number is used to identify the car and is associated with a time value once it's received by the data center. With the pressure of the red button the LED enlightens to indicate that the device is on;

- When the user comes back to the car he presses again the red button. At this moment the GPRS module transmits the registration number again and the time is associated to it again to calculate the exact permanence time and the LED turns off;

- The fare can be either directly withdrawn by the user's bank account or added to a monthly account.

This solution is evidently very simple because the interaction of the user is limited to the pressure of the red button. Moreover this solution also solves also the problems of the payment and of the parking period extension. Anyway this solution presents one big limitation deriving from the cost of the device which cannot be reduced under 50 Euros: this fact noteworthy influences its real utilization. 


\section{Other Applications}

\section{A. The Parking Operator Application}

As anticipated, while the user can choose one from the formerly described solutions, the parking operator will be simply provided with a PDA. A specific application has been developed for this device to allow the operator to check whether a user is paying for his parked car. The application has been realized using Java as a programming language and allows the operator also to impose fines and communicate directly with the database of the traffic police. The application is based on the following steps:

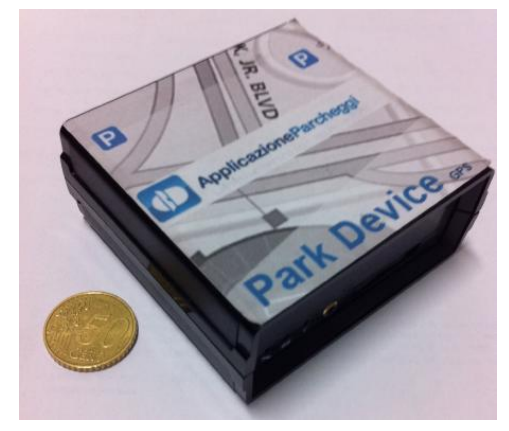

Figure 3. The GPS-GPRS box compared with a 50 Euro-cent coin

The operator writes the registration number of the car he wants to check inside a specific text field and he presses an "Enter" button;

- Then the operator selects the area where the car is parked;

- The system checks the presence of the registration number in the remote data center. If the number is found a page informs the operator;

- If the registration number is not found the operator is informed and he can choose to fine the user.

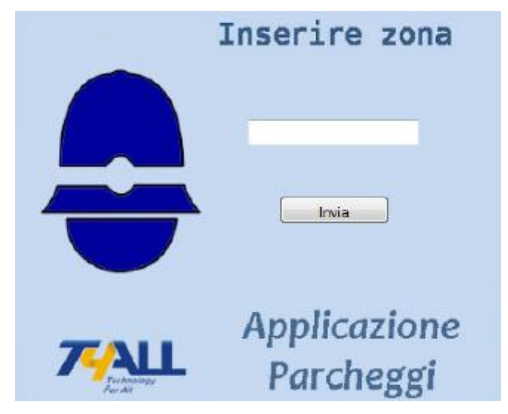

Figure 4. The Operator Home Page, with the text field for the identification number of the car

\section{B. The Internet Page}

Together with the applications for the parking operations also an Internet site has been set realized, to be used by both the users and the operators.

First of all the user has to choose in the Home page if he wants to log in as an end-user or as an operator: evidently the two sections of the site are separate and users can access only their section of the site.

The end-user section allows users to check if they are currently paying for some parking, to control all their past parking periods and to extend the parking period if they have paid for a parking in the traditional way.

The operator section is more complex and allows operators to manage all the information concerning the users.

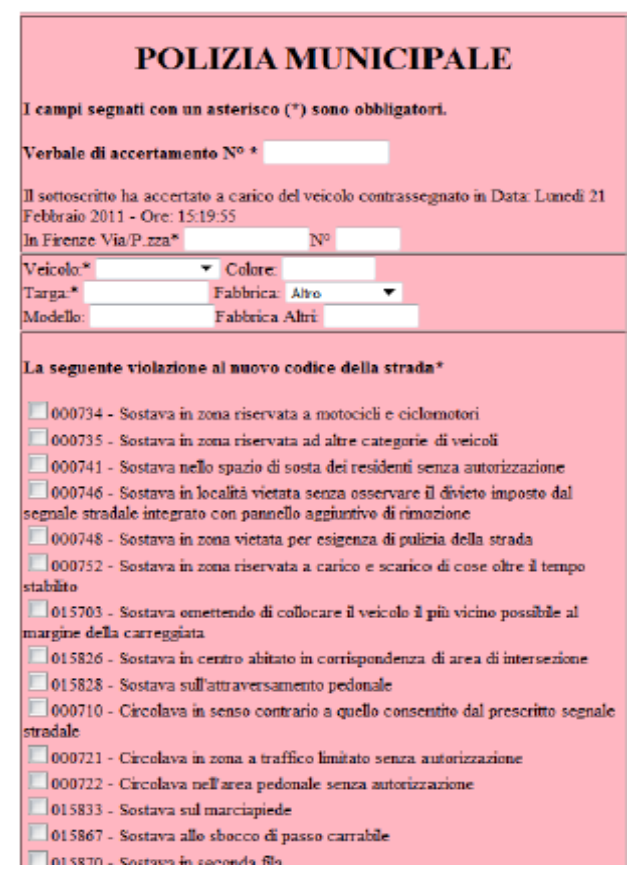

Figure 5. The electronic fine page

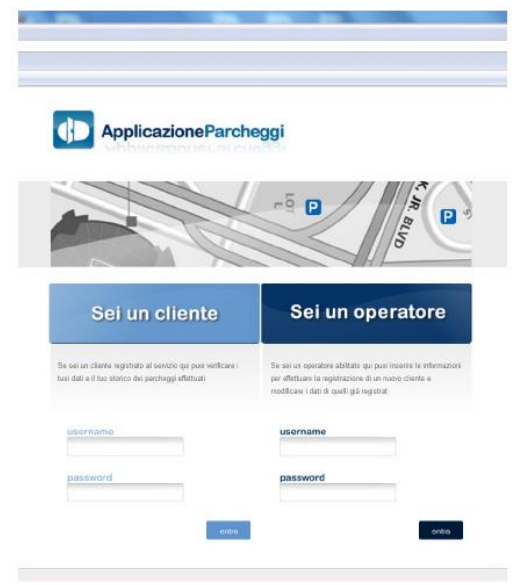

Figure 6. The Internet Home Page 
The operators can visualize the list of all the users or can perform specific researches if they know the Username, the First Name or the Family Name of a specific user. Once they have found the required user they can read all his personal data and all the information concerning the parking periods. Finally they can register new users.

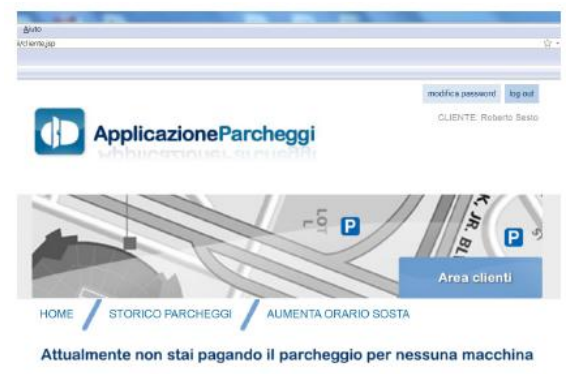

Figure 7. The end-user section

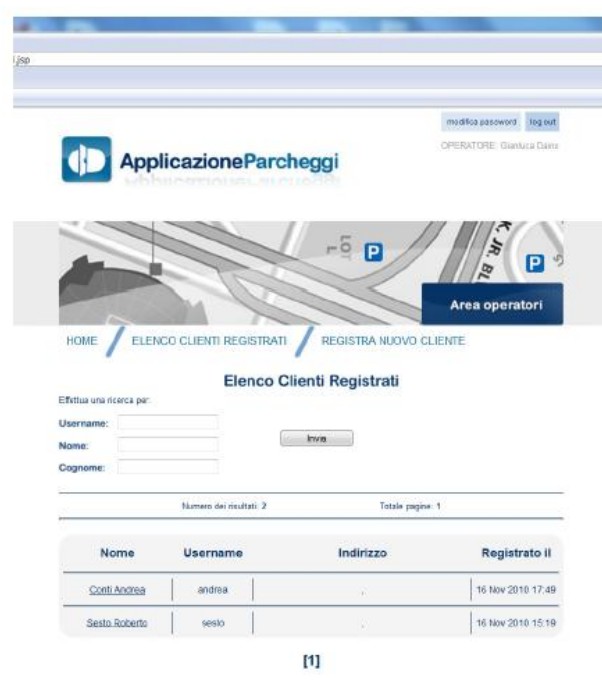

Figure 8. The operator section

\section{Conclusions}

The proposed solution appears to be interesting because it gives an answer to all the problems listed in the introduction. The closed car parks solution notably reduces the timing for two main factors: the user has not to go to the ticketing machine and when he enters and exits the car park the reading operation either of the NFC device or of the RFID Smart Card is notably faster that the collection and introduction of the standard parking tickets. This solution is also useful to speed up the payment operations because the user is not forced to search for the required amount of money. The solution for the street car parks also solves the underlined problems. The timing of the payment operation is in fact reduced with all the proposed solutions: while the solution using the GPS-GPRS module is evidently the fastest (only the time to press twice a button), also the other three significantly reduce the timing if compared with the traditional protocol. Anyway, the Mobile solution has the interaction with the phone as the delaying factor, while the NFC and RFID solutions are delayed by the necessity to go to the ticketing machine. The problem of the payment is solved in the same way by the four solutions because the payment of the parking is in all the cases performed remotely by bank transfer of by Credit Card.

The problem of the exact fare is solved in all the cases communicating both the beginning and ending times of the permanence, and then calculating the exact fare at the end of the permanence. All these solutions have then proven to be efficient when tested in laboratory. Anyway, the final test could only be done with a real implementation. For this purpose experimentation is going on in the city of Siena, even if currently this test only deals with the street car parks question and is based only on the development of the Mobile solution. Anyway, further experimentation is required for all the other contexts.

\section{References}

[1] NFC Forum, Near Field Communication and the NFC Forum: The Keys to Interoperable Communications, NFC Forum, 2007.

[2] Innovision, Near Field Communication in the Real World: Turning the NFC Promise into Profitable, Everyday Applications, Innovision Group, 2007.

[3] G. Benelli and A. Pozzebon, "An Automated Payment System for Car Parks Based On Near Field Communication Technology," Proceedings of ICITST 2010, The 5th International Conference fro Internet Technology and Secured Transactions, London, November 2010.

[4] K. Finkenzeller, RFID Handbook: Fundamentals and Applications in Contactless Smart Card and Identification, Wiley, 2003.

[5] M. Hanif, N. H. Hany, M. H. Badiozaman and D. Hanita, "Smart parking reservation system using short message services (SMS)," Proceedings of ICIAS 2010, International Conference on Intelligent and Advanced Systems, Kuala Lumpur, June 2010.

[6] R. R. Dholakia, M-commerce: global experiences and perspectives, IGI Global, 2006.

[7] Smart Card Alliance, Proximity Mobile Payments: Leveraging NFC and the Contactless Financial Payment Infrastructure, Smart Card Alliance, 2007. 